\title{
Effect of antagonistic salt on confined near-critical mixture
}

\author{
Faezeh Pousaneh ${ }^{1}$ and Alina Ciach $^{1}$ \\ ${ }^{1}$ Institute of Physical Chemistry, Polish Academy of Sciences, \\ Kasprzaka 44/52, PL-01-224 Warsaw, Poland
}

\begin{abstract}
We consider a near-critical binary mixture with addition of antagonistic salt confined between weakly charged and selective surfaces. A mesoscopic functional for this system is developed from a microscopic description by a systematic coarse-graining procedure. The functional reduces to the Landau-Brazovskii functional for amphiphilic systems for sufficiently large ratio between the correlation length in the critical binary mixture and the screening length. Our theoretical result agrees with the experimental observation [Sadakane et.al. J. Chem. Phys. 139, 234905 (2013)] that the antagonistic salt and surfactant both lead to a similar mesoscopic structure. For very small salt concentration $\rho_{\text {ion }}$ the Casimir potential is the same as in a presence of inorganic salt. For larger $\rho_{\text {ion }}$ the Casimir potential takes a minimum followed by a maximum for separations of order of tens of nanometers, and exhibits an oscillatory decay very close to the critical point. For separations of tens of nanometers the potential between surfaces with a linear size of hundreds of nanometers can be of order of $k_{B} T$. We have verified that in the experimentally studied samples [Sadakane et.al. J. Chem. Phys. 139, 234905 (2013), Leys et.al. Soft Matter 9, 9326 (2013)] the decay length is too small compared to the period of oscillations of the Casimir potential, but the oscillatory force could be observed closer to the critical point.
\end{abstract}




\section{INTRODUCTION}

Addition of small amount of salt to a binary mixture near a demixing critical point can significantly change its properties. When inorganic salt is added to water and organic

liquid, the two-phase region enlarges [1]. In contrast, antagonistic (or amphiphilic) salt added to such mixture leads to shrinking of the two phase region; it can even disappear when the amount of salt is large enough [2]. In addition, in the case of the antagonistic salt a peak in the structure factor for the wavenumber $k>0$ was observed in the onephase region [2 5]. The peak indicates thermodynamically stable inhomogeneities on the length scale $\sim 10 \mathrm{~nm}[2,5]$. Moreover, in a few cases a lamellar phase was observed for some region of the phase diagram [2, 3, 5]. For low salt concentration the shape of the structure factor was described with a good accuracy by the formula obtained by Onuki and Kitamura [6]. For larger amount of salt (when the two-phase region disappears) the experimental structure factor was described with better accuracy by the formula derived earlier for bicontinuous microemulsion or sponge phases [2, 7, 8]. In addition, the structure factor of the lamellar phase was fitted with a good accuracy [2] by the formula developed for a stack of membranes [2, 9]. These observations strongly suggest that the key features of the mesoscopic structure do not depend on whether antagonistic salt or surfactant is added to a mixture of inorganic and organic solvents. In this work we address the question of the above similarity on a theoretical level by comparing the Landau-type functionals for the two systems. For this purpose we first develop a Landau-type functional starting from a microscopic density functional theory and using the same coarse-graining procedure as used earlier for the hydrophilic salt [10, 11]. In the next step we verify under what conditions the Brazovskii functional of the solvent concentration, developed earlier for amphiphilic systems [12, 13, 15] can be obtained.

The effects of confinement on the near-critical mixture with antagonistic salt were not investigated experimentally yet. Theoretical investigations based on the theory of Onuki and Kitamura [6] focused very briefly on colloid particles immersed in the critical mixture with salt [16]. In contrast, the effective potentials between a flat substrate and a colloid particle immersed in the critical mixture with hydrophilic salt, and between two particles in such mixture were measured in several impressive experiments [17 21]. Similarity between the structure factors in the investigated system and sponge phases suggests that an oscillatory 
force between confining surfaces, observed experimentally for surfactant mixtures [14], could occur in a presence of antagonistic salt too.

The effective potential between surfaces confining the critical mixture with ions is interesting from both the fundamental and the practical point of view. Surfaces with similar adsorption preferences attract each other, while surfaces with different adsorption preferences repel each other [19, 22 24] when the confined fluid is near its critical point. The range of this so called thermodynamic Casimir potential [19, 24] is equal to the bulk correlation length $\xi \propto\left|T-T_{c}\right|^{-\nu}$ with $\nu \approx 0.63$, and becomes macroscopic when the critical temperature $T_{c}$ is approached. When the surfaces are charged, then depending on the surface charges electrostatic repulsion or attraction is added to the Casimir potential. The resulting potential can have a minimum or a maximum, depending on the ratio between $\xi$ and the screening length $\lambda_{D}$. By changing the salt concentration and the temperature, one can change $\lambda_{D}$ and $\xi$, and tune the shape of the potential between the surfaces, e.g. the surfaces of particles $[17-20]$. Temperature can be changed in a reversible manner, and reversible structural changes can be induced [20, 21, 25]. In particular, when the effective potential has a minimum, then analogs of the gas-liquid and liquid-solid transitions between the particles were observed [20].

The experimental results for inorganic salt show that the effective potential cannot be described by just a sum of the Casimir and the electrostatic potential [19, 26]. In particular, attraction was measured for some temperature range between like charge hydrophilic and hydrophobic surfaces [18], whereas both the Casimir and the electrostatic potentials are repulsive in this case. Both potentials, however, can be modified because of different solubility of the ions in the two components of the solvent. For this reason quite different behavior of the effective potential between charged and selective surfaces can be expected for the antagonistic salt [16], and we address this question here.

Several groups tried to explain the experiment in Ref. [18]. The attraction for a range of temperatures was obtained in two different approaches. The first one is based on the theory of Onuki and Kitamura [6]. The attraction appears when the solubility in water of the anion and the cation differ significantly from each other and the hydrophobic surface is neutral [27, 28]. On the other hand, in the theory developed in Ref.[10] for hydrophilic ions, the concentration profile near the charged hydrophobic surface can be nonmonotonic, because the attraction of the organic particles competes with the attraction of the hydrated 
ions [11, 29]. When $\lambda_{D}>\xi$, excess of water may appear at some distance from the surface, therefore it may act as a hydrophilic one. The shapes of the effective potential for different temperatures in this theory are quite similar to the experimental curves, but the fitting was not attempted yet [26]. Importantly, good quantitative agreement between this theory and the experiments conducted for $\lambda_{D}<\xi$ [17, 19] was obtained in Ref.[30], in contrast to the theories based on the Onuki and Kitamura functional. In this work we develop a Landau-type theory for the antagonistic salt following the same strategy as in Ref.[10, 11].

In the Onuki and Kitamura model the critical binary mixture is described by the phenomoenological Landau functional of the concentration $\phi$. The ions are treated as a twocomponent ideal gas whose particles interact with the Coulomb potential, and the corresponding entropy and electrostatic energy are added to the Landau functional. Finally, the coupling between the critical mixture and the ions of the form $\phi\left(w_{+} n_{+}+w_{-} n_{-}\right)$is added, where $n_{i}$ and $w_{i}$ denote the density and the preferential salvation of the $i$-th ion. Note that in this model the van der Waals interactions between the ions are neglected, and the entropy of the four-component mixture is approximated by a sum of the entropy of the two twocomponent subsystems. For this reason the coupling between the two subsystems beyond the above bilinear term is not taken into account.

The full microscopic density-functional theory of a four-component mixture is more accurate and justified than a phenomenological model, but in practice it is too difficult. Moreover, such a detailed description is not necessary when the characteristic length scales are mesoscopic. On the other hand, a microscopic theory is a good starting point for a derivation of the Landau-type functional by a coarse-graining procedure. Such a strategy was successful in the case of the hydrophilic salt [10, 26]. In the next section we develop in the same way a Landau-type functional for the antagonistic salt added to the near-critical binary solvent. In sec. 3 we focus on the disordered phase in the bulk. We calculate the correlation function, and compare our functional with the Landau-Brazovskii functional for microemulsions. In sec. 4 we consider the concentration and charge profiles in a semiinfinite system and in a slit, and calculate the effective potential between the surfaces. In sec. 5 a comparison of the structure factor with the experimental results reported in Ref. [2, 5] is performed. For the model parameters obtained from the fitting of the structure factor to the experimental results the concentration profiles and the effective potential are computed. Sec.6 contains summary and discussion. In particular, we compare the effective potential obtained in the 
models for the hydrophilic and the antagonistic salt.

\section{THE MODEL}

We consider a four-component mixture containing water, organic liquid and antagonistic salt which has hydrophilic cations and hydrophobic anions. The fluid is in contact with a reservoir with fixed temperature and chemical potential of all the components. In equilibrium the distribution of the components corresponds to the minimum of the grand potential

$$
\begin{aligned}
\Omega & =-P V \\
& =U_{v d W}+U_{e l}-T S-\int_{V} d \mathbf{r}^{*} \mu_{i} \rho_{i}^{*}(\mathbf{r}),
\end{aligned}
$$

where $P, V$ and $S$ are pressure, volume and entropy respectively, and $U_{e l}, U_{v d W}$ are the electrostatic and the van der Waals contributions to the internal energy. $\rho_{i}(\mathbf{r})$ and $\mu_{i}$ are the local number density and the chemical potential of the $i$-th component respectively, with $i=w, l$ corresponding to water and organic solvent (for example lutidine or methylpyridine), and $i=+,-$ corresponding to the cations and the anions. We consider dimensionless distance $r^{*}=r / a$ and dimensionless densities, i.e. the length is measured in units of $a$, and $\rho_{i}^{*}=a^{3} N_{i} / V$, where $a^{3}$ is the average volume per particle in the liquid phase, and $N_{i}$ denotes the number of the $i$-th kind particles in the volume $V$. We neglect compressibility of the liquid and assume that the total density is fixed,

$$
\sum_{i=\{w, l,+,-\}} \rho_{i}^{*}=1
$$

The microscopic details, in particular different sizes of molecules are disregarded, since we are interested in the local densities in the regions much larger than $a$. To simplify the notation we shall omit the asterisk for the dimensionless distance.

The electrostatic energy is given by [31]

$$
U_{e l}=\int_{V} d \mathbf{r}\left[-\frac{\epsilon}{8 \pi}\left(\nabla \psi_{e l}(\mathbf{r})\right)^{2}+e \rho_{q}^{*}(\mathbf{r}) \psi_{e l}(\mathbf{r})\right]
$$

where $\psi_{e l}(\mathbf{r})$ is the electrostatic potential which satisfies the Poisson equation (note that the length $|\mathbf{r}|$ and density are dimensionless)

$$
\nabla^{2} \psi_{e l}(\mathbf{r})=-\frac{4 \pi e}{a \epsilon} \rho_{q}^{*}(\mathbf{r})
$$


We neglect the dependence of the dielectric constant $\epsilon$ on the solvent concentration $([10,11])$. $\rho_{q}^{*}(\mathbf{r})$ is the local dimensionless charge density

$$
\rho_{q}^{*}(\mathbf{r})=\rho_{+}^{*}(\mathbf{r})-\rho_{-}^{*}(\mathbf{r})
$$

and $e$ is the elementary charge.

We assume the usual form of the internal energy $\mathcal{U}_{v d W}$,

$$
\mathcal{U}_{v d W}=\int_{V} d \mathbf{r} \int_{V} d \mathbf{r}^{\prime} \frac{1}{2} \rho_{i}^{*}(\mathbf{r}) V_{i j}\left(\mathbf{r}-\mathbf{r}^{\prime}\right) g_{i j}\left(\mathbf{r}-\mathbf{r}^{\prime}\right) \rho_{j}^{*}\left(\mathbf{r}^{\prime}\right),
$$

where $V_{i j}$ and $g_{i j}$ are the vdW interaction and the pair correlation function between the corresponding components respectively. The very complex expression for $\mathcal{U}_{v d W}$ can be approximated by a much simpler form in systems with particular properties of $V_{i j}$, and in special thermodynamic states, such as the neighborhood of the critical point. Close to the critical point and for small amount of ions the correlation and screening lengths are large, and the local densities vary slowly on the microscopic length scale. Thus, the density $\rho_{j}^{*}\left(\mathbf{r}^{\prime}\right)$ can be Taylor expanded about $\mathbf{r}$. In the case of attractive interactions the expansion can be truncated at the second-order therm [10, 32]. As a result, $\mathcal{U}_{v d W}$ is given by a single integral over $\mathbf{r}$ with the integrand depending on the densities and their gradients at $\mathbf{r}$, and on the zeroth, $J_{i j}=-\int_{V} d \mathbf{r} V_{i j}(\mathbf{r}) g_{i j}(\mathbf{r})$, and the second, $\bar{J}_{i j}=-\int_{V} d \mathbf{r} V_{i j}(\mathbf{r}) g_{i j}(\mathbf{r}) r^{2} / 6$, moments of $V_{i j} g_{i j}[10$, 32]. Since we consider two inorganic (water and cation) and two organic (metylpyridine and anion) components, we can further simplify the expression for $U_{v d W}$ by assuming appropriate relations between $J_{i j}$. We assume that the differences between the interactions of all the inorganic components are negligible; likewise, we neglect the differences between the interactions of all the organic components. Accordingly, we assume

$$
\begin{array}{r}
J_{w w} \approx J_{++} \approx J_{w+} \\
J_{l l} \approx J_{--} \approx J_{l-} \\
J_{w l} \approx J_{w-} \approx J_{l+} \approx J_{+-} .
\end{array}
$$

with analogous assumptions for $\bar{J}_{i j}$. After some algebra we obtain

$$
\mathcal{U}_{v d W}=U_{v d W}[\Phi]+J_{1} \int_{V} \Phi(\mathbf{r}) d \mathbf{r}+J_{0} V
$$


where

$$
U_{v d W}[\Phi]=\frac{J}{2} \int_{V}\left[-b \Phi(\mathbf{r})^{2}+(\nabla \Phi(\mathbf{r}))^{2}\right] d \mathbf{r}
$$

and

$$
\Phi(\mathbf{r})=\rho_{w}^{*}(\mathbf{r})-\rho_{l}^{*}(\mathbf{r})+\rho_{q}^{*}(\mathbf{r})
$$

is the difference between the local dimensionless density of the inorganic and organic components (see (5) $), J_{1}=\frac{1}{4}\left(J_{w w}-J_{l l}\right), J_{0}=\frac{1}{8}\left(J_{w w}+J_{l l}+2 J_{w l}\right)$, and

$$
J=\frac{1}{4}\left(\bar{J}_{w w}+\bar{J}_{l l}-2 \bar{J}_{w l}\right)
$$

is the energy parameter relevant for the phase separation. The parameter $b$ is associated with the difference between the second and the zeroth moments of the interactions. In the case of the lattice model with nearest-neighbor interactions $b=2 d$ in a $d$-dimensional system. We neglect the constant term in (8), and add the linear term in (8) to $-\mu_{i} \sum_{i} \rho_{i}^{*}(\mathbf{r})$ in Eq.(11). As a consequence, the internal energy is given in Eq.(99) and the chemical potentials are modified.

Finally, we postulate the lattice gas or ideal-mixing form for the entropy

$$
-T S=k_{B} T \int_{V} d \mathbf{r} \sum_{\{i=w, l,+,-\}} \rho_{i}^{*}(\mathbf{r}) \ln \rho_{i}^{*}(\mathbf{r}) .
$$

Eq. (12) should be expressed in terms of the new variables $\Phi, \rho_{q}^{*}$ and the dimensionless ion density

$$
\rho_{i o n}^{*}(\mathbf{r})=\rho_{+}^{*}(\mathbf{r})+\rho_{-}^{*}(\mathbf{r}),
$$

since there are 3 independent variables when (2) holds.

In this theory the thermal equilibrium for fixed temperature, total density and chemical potentials corresponds to the global minimum of the grand potential (1) with the electrostatic and the vdW contributions to the internal energy given in Eqs.(3) and (9) respectively, and the entropy given in (12). The equilibrium forms of $\Phi(\mathbf{r})$ and $\rho_{i o n}^{*}(\mathbf{r})$ are the solutions of the Euler-Lagrange (EL) equations obtained from the extremum conditions $\delta \Omega / \delta \rho_{i o n}^{*}(\mathbf{r})=$ $0=\delta \Omega / \delta \Phi(\mathbf{r})=0=\delta \Omega / \delta \rho_{q}^{*}(\mathbf{r})$. Note that neither $U_{\text {el }}$ nor $U_{v d W}$ depends on $\rho_{i o n}^{*}(\mathbf{r})$. For this reason $\rho_{i o n}^{*}(\mathbf{r})$ can be easily expressed in terms of $\Phi(\mathbf{r})$ and $\rho_{q}^{*}(\mathbf{r})$ with the help of the equation $\delta \Omega / \delta \rho_{i o n}^{*}(\mathbf{r})=0$ (see Appendix A), and the grand potential becomes a functional of two fields, $\Phi(\mathbf{r})$ and $\rho_{q}^{*}(\mathbf{r})$. 


\section{PROPERTIES OF THE DISORDERED PHASE}

\section{A. Landau-type functional in the Gaussian approximation}

In thermodynamic conditions corresponding to stability of a uniform fluid $\rho_{q}^{*}(\mathbf{r})=0$ and both $\Phi(\mathbf{r})$ and $\rho_{i o n}^{*}(\mathbf{r})$ are independent of the space position, $\Phi(\mathbf{r})=\bar{\Phi}$ and $\rho_{i o n}^{*}(\mathbf{r})=\bar{\rho}_{i o n}^{*}$. For the stability analysis and in order to calculate the correlation functions we introduce the Landau-Ginzburg (LG) functional

$$
\mathcal{L}\left[\Phi(\mathbf{r}), \rho_{q}^{*}(\mathbf{r})\right]=\beta \Omega\left[\Phi(\mathbf{r}), \rho_{i o n}^{*}(\mathbf{r}), \rho_{q}^{*}(\mathbf{r})\right]-\beta \Omega\left[\bar{\Phi}, \bar{\rho}_{i o n}^{*}, 0\right],
$$

where $\rho_{\text {ion }}^{*}(\mathbf{r})$ is expressed in terms of $\Phi(\mathbf{r})$ and $\rho_{q}^{*}(\mathbf{r})$ (Appendix A) and

$$
\Phi(\mathbf{r})=\Phi(\mathbf{r})-\bar{\Phi}
$$

We write the internal energy in the Fourier representation (Appendix B). Next we Taylorexpand the entropy minimized with respect to $\rho_{i o n}^{*}$ and approximate it by a second-order polynomial in $\Phi$ and $\rho_{q}^{*}$. This way we obtain the LG functional in the Gaussian approximation,

$\mathcal{L}_{G}[\Phi(\mathbf{k}), \rho(\mathbf{k})]=\frac{1}{2} \int_{V} \frac{d \mathbf{k}}{(2 \pi)^{d}}\left\{\tilde{C}_{\Phi \Phi}(k) \tilde{\Phi}(\mathbf{k}) \tilde{\Phi}(-\mathbf{k})+2 \tilde{C}_{\Phi q}(k) \tilde{\Phi}(\mathbf{k}) \tilde{\rho}_{q}^{*}(-\mathbf{k})+\tilde{C}_{q q}(k) \tilde{\rho}_{q}^{*}(\mathbf{k}) \tilde{\rho}_{q}^{*}(-\mathbf{k})\right\}$,

where

$$
\left\{\begin{array}{l}
\tilde{C}_{\Phi \Phi}(k)=\beta^{*}\left(\xi_{0}^{-2}+k^{2}\right), \\
\tilde{C}_{\Phi q}(k)=-\frac{1}{\left(1-\bar{\rho}_{i o n}^{*}-\bar{\Phi}^{2}\right)}, \\
\tilde{C}_{q q}(k)=\frac{1}{\bar{\rho}_{i o n}^{*}}+\frac{1}{\left(1-\bar{\rho}_{i o n}^{*}-\bar{\Phi}^{2}\right)}+\frac{1}{\bar{\rho}_{i o n}^{*} \lambda_{D}^{* 2} k^{2}},
\end{array}\right.
$$

with $1 / \beta^{*}=T^{*}=k_{B} T / J$, the dimensionless screening length $\lambda_{D}^{*}$ given by

$$
\lambda_{D}^{* 2}=\lambda_{D}^{2} / a^{2}=\frac{a \epsilon}{4 \pi e^{2} \beta \bar{\rho}_{\text {ion }}^{*}}
$$

and with

$$
\xi_{0}=\left(\frac{T^{*}}{\left(1-\bar{\rho}_{i o n}^{*}-\bar{\Phi}^{2}\right)}-b\right)^{-1 / 2} .
$$

For $\tilde{\rho}_{q}^{*}(\mathbf{k})=0$ Eq.(16) reduces to the standard Landau functional for the upper critical point. $\xi_{0}$ is the dimensionless correlation length in the case of $\tilde{\rho}_{q}^{*}(\mathbf{k})=0$ and $T^{*}>T_{c}^{*}$, where 
$T_{c}^{*}=b\left(1-\bar{\rho}_{\text {ion }}^{*}\right)$ is the critical temperature in the reduced units. $\bar{\Phi}=0$ at the critical point. The temperature of the upper critical point decreases after addition of the solute, because the entropy increases, and the energy is not changed in this model (see (7)). In the case of the lower critical point $T^{*}-T_{c}^{*}$ in (19) should be replaced by $\left|T^{*}-T_{c}^{*}\right|$.

\section{B. The structure and the boundary of stability of the disordered phase}

According to the density functional theory [33] the correlation function for concentration fluctuations, $\tilde{G}(k)$ is given by

$$
\tilde{G}(k)=\frac{\tilde{C}_{q q}(k)}{\tilde{C}_{\Phi \Phi}(k) \tilde{C}_{q q}(k)-\tilde{C}_{\Phi q}(k)^{2}},
$$

and its explicit form can be easily obtained from Eq. (17),

$$
\tilde{G}(k)=\xi_{0}^{2} T^{*}\left\{1+\xi_{0}^{2} k^{2}-\frac{a_{N} \xi_{0}^{2} k^{2}}{\lambda_{D}^{* 2} k^{2}+a_{D}}\right\}^{-1} .
$$

We have introduced

$$
a_{N}=\frac{T^{*} \bar{\rho}_{i o n}^{*} \lambda_{D}^{* 2}}{\left(1-\bar{\rho}_{i o n}^{*}-\bar{\Phi}^{2}\right)\left(1-\bar{\Phi}^{2}\right)}
$$

and

$$
a_{D}=\frac{\left(1-\bar{\rho}_{\text {ion }}^{*}-\bar{\Phi}^{2}\right)}{\left(1-\bar{\Phi}^{2}\right)} .
$$

Our formula (21) is very similar to the one obtained in Ref.[6]. The $k$-dependence is the same, but in Ref.[6] $a_{D}=1$, and our expression for $a_{N}$ is somewhat different. We should stress that in our theory $\xi_{0}$ is equal to the correlation length in the system with suppressed charge waves.

Note that $\mathcal{L}_{G}\left[\Phi, \rho_{q}\right]$ in Eq.(16) can be minimized with respect to $\rho_{q}^{*}$, because $\tilde{C}_{q q}(k)>0$. At the minimum $\tilde{\rho}_{q}^{*}(\mathbf{k})=-\frac{\tilde{C}_{\Phi q}(k)}{\tilde{C}_{q q}(k)} \tilde{\Phi}(\mathbf{k})$, and the functional takes the form

$$
\mathcal{L}_{G}[\Phi]=\frac{1}{2} \int_{V} \frac{d \mathbf{k}}{(2 \pi)^{d}} \tilde{C}(k) \tilde{\Phi}(\mathbf{k}) \tilde{\Phi}(-\mathbf{k})
$$

where (see (21) $)$

$$
\tilde{C}(k)=\tilde{G}^{-1}(k)
$$

$\tilde{C}(k)$ takes the minimum

$$
\tilde{C}\left(k_{0}\right)=\beta^{*}\left[\left(\frac{\lambda_{D}^{*}}{\xi_{0}}\right)^{2}-\left(\sqrt{a}_{N}-\sqrt{a}_{D}\right)^{2}\right] \lambda_{D}^{*-2}
$$


for $k=k_{0}$ with

$$
k_{0}^{2} \lambda_{D}^{* 2}=\sqrt{a_{D}}\left(\sqrt{a_{N}}-\sqrt{a_{D}}\right)
$$

where $a_{N}$ and $a_{D}$ are given in (22) and (23). When $a_{N}<a_{D}$, then $\tilde{C}(k)$ takes the minimum at $k=0$. The minimum of $\tilde{C}(k)$ (or the maximum of $\tilde{G}(k)$ ) with $k_{0}>0$ occurs only if $a_{N}>a_{D}$, or explicitly

$$
\left(k_{B} T\right)^{2}>J \frac{4 \pi e^{2}}{a \epsilon}\left(1-\bar{\rho}_{i o n}^{*}-\bar{\Phi}^{2}\right)^{2} .
$$

In our theory developed for an upper critical point the inhomogeneous structure in the disordered phase can occur when the thermal energy is larger than the geometric mean of the energy cost of the salt molecule dissociation and the vdW energy gain of phaseseparated system, $J$. At the same time $J$ is the energy cost of a local interface (see the term $\frac{J}{2} \int d \mathbf{r}(\nabla \Phi)^{2}$ in Eq.(99)), and $4 \pi e^{2} /(a \epsilon)$ is the energy gain when the two ions approach each other from the two sides of the interface.

The correlation function in the real-space representation can be obtained by the pole analysis of $\tilde{G}(k)$. We have found that the damped oscillatory and the monotonic decay of correlations occur for $\left(\sqrt{a}_{N}-\sqrt{a}_{D}\right)^{2}<\left(\lambda_{D}^{*} / \xi_{0}\right)^{2}<\left(\sqrt{a}_{N}+\sqrt{a}_{D}\right)^{2}$, and $\left(\lambda_{D}^{*} / \xi_{0}\right)>\left(\sqrt{a}_{N}+\right.$ $\left.\sqrt{a}_{D}\right)$ respectively. The boundary between these two cases, $\left(\lambda_{D}^{*} / \xi_{0}\right)=\left(\sqrt{a}_{N}+\sqrt{a}_{D}\right)$ is called the disorder surface in the $\left(T^{*}, \bar{\rho}_{i o n}^{*}, \bar{\Phi}\right)$ phase diagram, as in the amphiphilic systems [13].

When $\left(\sqrt{a}_{N}-\sqrt{a}_{D}\right)=\left(\lambda_{D}^{*} / \xi_{0}\right)$ then $\tilde{C}\left(k_{0}\right)=0$ (i.e. $\tilde{G}\left(k_{0}\right) \rightarrow \infty$ ), and the homogeneous phase is at the boundary of stability in the mean-field approximation (MF). At the corresponding $\lambda$ - surface in the $\left(T^{*}, \bar{\rho}_{i o n}^{*}, \bar{\Phi}\right)$ phase space (we use this name by analogy with the $\lambda$-line [34] in the two-dimensional phase space) $k_{0}$ is given by

$$
k_{0}^{2} \lambda_{D}^{*} \xi_{0}=\sqrt{a}_{D} \approx 1
$$

The wavelength of the concentration wave at the $\lambda$ - surface is equal to a geometric mean of the inverse correlation length and the inverse screening length. This relation allows for a quick verification if mesoscopic inhomogeneities $\left(k_{0} \sim 2 \pi / 10 \mathrm{~nm}^{-1}\right)$ can occur in the investigated experimental system. 


\section{Comparison with microemulsions}

When we limit ourselves to the most probable concentration waves, with the wavenumbers near the maximum of the structure factor, $k \simeq k_{0}>0$, then we can make the approximation

$$
\tilde{C}(k) \simeq \tilde{C}\left(k_{0}\right)+c\left(k^{2}-k_{0}^{2}\right)^{2} .
$$

We took into account that $\tilde{C}(k)$ is a function of $k^{2}$, and truncated the Taylor expansion in $k^{2}$ about $k_{0}^{2}$ at the second-order term. After inserting (30) in (24) we obtain the LandauBrazovskii functional [15] in the Gaussian approximation

$$
\mathcal{L}_{G}[\Phi] \approx \frac{c}{2} \int_{V} \frac{d \mathbf{k}}{(2 \pi)^{d}} \tilde{\Phi}(\mathbf{k})\left[t_{0}+\left(k^{2}-k_{0}^{2}\right)^{2}\right] \tilde{\Phi}(-\mathbf{k})
$$

where $k_{0}$ is given in (27),

$$
t_{0}=\tilde{C}\left(k_{0}\right) / c
$$

$\tilde{C}\left(k_{0}\right)$ is given in (26) and $c=\beta^{*} \lambda_{D}^{* 2} / \sqrt{a_{N} a_{D}}$. The Landau-Brazovskii functional was successfully used for a description of the structure of block copolymers [35], binary or ternary surfactant mixtures [12, 36] and recently for the colloidal self-assembly [32]. In each case the physical interpretation of the order-parameter $\Phi$ is different. In particular, in the case of ternary surfactant mixtures $\Phi$ is interpreted as a concentration difference between the polar and nonpolar components, in close analogy with the present case where $\Phi$ is a concentration difference between the inorganic and organic components.

From Eq.(30) we can easily obtain the approximate expression for the correlation function in the real-space representation [13],

$$
G(r)=\frac{\lambda}{2 \pi r} e^{-r / \xi} \sin \left(\frac{2 \pi r}{\lambda}\right),
$$

where the dimensionless correlation length and period of the exponentially damped oscillatory decay, $\xi$ and $\lambda$, are given by

$$
\xi^{2}=\frac{2}{\sqrt{t_{0}+k_{0}^{4}}-k_{0}^{2}}
$$

and

$$
\left(\frac{\lambda}{2 \pi}\right)^{2}=\frac{2}{\sqrt{t_{0}+k_{0}^{4}}+k_{0}^{2}} .
$$

Note that $\xi$ differs from $\xi_{0}$ and diverges at the boundary of stability of the disordered phase, $t_{0}=0$. Moreover, $\lambda \leq 2 \pi / k_{0}$; the equality holds when $t_{0}=0$. 
We should mention that in the Brazovskii theory the dominant fluctuations $\left(k \simeq k_{0}\right)$ lead to fluctuation-induced first-order phase transition to the lamellar phase beyond the Gaussian approximation [15], or as shown recently to a transition to a nematic phase [38]. The $k$ dependence of $\tilde{G}(k)$ in the disordered phase remains almost unchanged beyond the Gaussian approximation, however, but $t_{0}$, and hence the model parameters, become renormalized [15]. For this reason Eq.(21) can reasonably well describe the experimental results, but the MF theories cannot correctly predict the microscopic expressions for the parameters present in Eq.(21).

The similarity between our system and ternary surfactant mixtures was confirmed by the agreement of the experimental results for the former and the formulas developed for the latter case [2]. Here we have shown that starting from a simple microscopic density functional theory we can obtain by a systematic coarse-graining procedure the same Landau-Brazovskii functional (31) that describes the amphiphilic systems.

\section{EFFECTS OF CONFINEMENT}

\section{A. The Euler-Lagrange equations}

In order to obtain the form of $\Phi$ and $\rho_{q}^{*}$ it is necessary to solve the EL equations for $\Phi, \psi$, $\rho_{i o n}^{*}$ and $\rho_{q}^{*}$. The expressions corresponding to the full functional (11) with the electrostatic and the vdW contributions to the internal energy given in Eqs.(3) and (9) respectively, and the entropy given in (12) are rather long, and are given in Appendix A. Here we discuss the linearized EL equations for $\bar{\Phi}=0$ (see (66) and (67) in the Appendix A) that can be written in the form

$$
\nabla^{2} \nabla^{2} \Phi(\mathbf{r})+a_{2} \nabla^{2} \Phi(\mathbf{r})+a_{0} \Phi(\mathbf{r})=0
$$

and

$$
\rho_{q}^{*}(z)=\frac{\left(1-\bar{\rho}_{i o n}^{*}\right)}{T^{*}}\left[\xi_{0}^{-2} \Phi(z)-\nabla^{2} \Phi(z)\right],
$$

where $\nabla^{2}$ denotes the laplacian,

$$
a_{2}=\left[\left(a_{N}-a_{D}\right)-\lambda_{D}^{* 2} \xi_{0}^{-2}\right] \lambda_{D}^{*-2},
$$

and

$$
a_{0}=a_{D} \xi_{0}^{-2} \lambda_{D}^{*-2}
$$


Let us focus for simplicity on one-dimensional concentration profiles. The solution of (36) for $\Phi$ depending only on $z$ is a linear combination of the exponential terms $\exp \left( \pm \lambda_{i} z\right)$ with

$$
\lambda_{1,2}^{2}=\frac{-a_{2} \pm \sqrt{\Delta}}{2}
$$

where $\Delta$ can be written in the form

$$
\Delta=\left[\lambda_{D}^{* 2} \xi_{0}^{-2}-\left(\sqrt{a}_{N}-\sqrt{a}_{D}\right)^{2}\right]\left[\lambda_{D}^{* 2} \xi_{0}^{-2}-\left(\sqrt{a}_{N}+\sqrt{a}_{D}\right)^{2}\right] \lambda_{D}^{*-4}
$$

Note that $\Delta=0$ when either the first or the second factor in (41) vanishes. The first factor in (41) is proportional to $t_{0}$ (see (32)), hence $\Delta$ vanishes at the $\lambda$-surface $t_{0}=0$. The second factor vanishes at the disorder surface $\lambda_{D}^{*} / \xi_{0}=\left(\sqrt{a}_{N}+\sqrt{a}_{D}\right)$. For $\left(\sqrt{a}_{N}-\sqrt{a}_{D}\right)^{2}<$ $\left(\lambda_{D}^{*} / \xi_{0}\right)^{2}<\left(\sqrt{a}_{N}+\sqrt{a}_{D}\right)^{2}$ the $\lambda_{i}$ are complex conjugate numbers, because $\Delta<0$. For $\lambda_{D}^{*} / \xi_{0}>\left(\sqrt{a}_{N}+\sqrt{a}_{D}\right)$ both $\lambda_{i}$ are real, while for $\left(\lambda_{D}^{*} / \xi_{0}\right)^{2}<\left(\sqrt{a}_{N}-\sqrt{a}_{D}\right)^{2}\left(\right.$ i.e. $\left.t_{0}<0\right)$ both $\lambda_{i}$ are imaginary. Note that for $t_{0}=0$ we obtain an oscillatory function with the wavenumber of oscillations equal to $k_{0}$, in consistency with the results of the previous section.

\section{B. The boundary conditions for the EL equations}

When the system is in contact with a selective and charged surface at $z=0$, then there is additional contribution to the internal energy [10, 11, 30]

$$
U_{s}=\left[J\left(\frac{\Phi^{2}(0)}{2}-h_{0} \Phi(0)\right)+e \sigma_{0} \psi(0)\right] A^{*}
$$

where $A^{*}$ is the dimensionless area of the confining surface, $\sigma_{0}$ is the dimensionless surface charge (the charge per area $a^{2}$ ), $h_{0}$ is the dimensionless surface field describing the preferential adsorption of the inorganic (for $h_{0}>0$ ) or organic (for $h_{0}<0$ ) components, and the first term follows from the missing fluid neighbors for $z \leq 0$. In the case of a slit with another surface at $z=L$ there is analogous contribution to the internal energy. The above surface terms lead to the boundary conditions for the EL equations [10, 11]

$$
\nabla \Phi(0)-\Phi(0)=-h_{0}, \quad \nabla \Phi(L)+\Phi(L)=h_{L}
$$

and

$$
\nabla \psi(0)=-\frac{4 \pi e}{\epsilon} \sigma_{0}, \quad \nabla \psi(L)=\frac{4 \pi e}{\epsilon} \sigma_{L}
$$


In the linearized theory

$$
\psi(\mathbf{r})=\frac{k_{B} T}{e\left(1-\bar{\rho}_{i o n}^{*}\right)}\left[\Phi(\mathbf{r})-\frac{\rho_{q}^{*}(\mathbf{r})}{\bar{\rho}_{i o n}^{*}}\right]
$$

when $\bar{\Phi}=0$, and from (37) we obtain using (44) the second boundary conditions for the equation (36)

$$
\Phi^{\prime \prime \prime}(0)-\zeta^{-2} \Phi^{\prime}(0)=-\frac{T^{*}}{\lambda_{D}^{* 2}} \sigma_{0}, \quad \Phi^{\prime \prime \prime}(L)-\zeta^{-2} \Phi^{\prime}(L)=\frac{T^{*}}{\lambda_{D}^{* 2}} \sigma_{L}
$$

where

$$
\zeta^{-2}=\xi_{0}^{-2}-\frac{T^{*} \bar{\rho}_{i o n}^{*}}{1-\bar{\rho}_{i o n}^{*}}
$$

\section{The local concentration and the local charge density in a semiinfinite system}

We shall limit ourselves to a near-surface structure in the disordered phase and to the critical composition $\bar{\Phi}=0$. The composition of the near-surface layer depends on both the wall-fluid van der Waals interactions $h_{0}$ and on the sign and value of the surface charge $\sigma_{0}$. A hydrophilic surface attracts water, and a negatively charged surface attracts water-soluble ions. In contrast, the positively charged surface attracts ions soluble in the organic solvent, and in the case of the positively charged hydrophilic surface the composition in its vicinity depends on the ratio $h_{0} / \sigma_{0}$. We shall limit ourselves to $h_{0}>0$.

Let us first focus on the case of $\lambda_{D}^{*} / \xi_{0}>\left(\sqrt{a}_{N}+\sqrt{a}_{D}\right)$, where both $\lambda_{i}$ are real numbers. From (36) and (37) we have

$$
\Phi(z)=A_{1} e^{-\lambda_{1} z}+A_{2} e^{-\lambda_{2} z}
$$

and

$$
\rho_{q}^{*}(z)=\frac{\left(1-\bar{\rho}_{\text {ion }}^{*}\right)}{T^{*}}\left[\left(\xi_{0}^{-2}-\lambda_{1}^{2}\right) A_{1} e^{-\lambda_{1} z}+\left(\xi_{0}^{-2}-\lambda_{2}^{2}\right) A_{2} e^{-\lambda_{2} z}\right]
$$

where $A_{i}$ are determined by the boundary conditions and depend on $\sigma_{0}$ and $h_{0}$ (see Appendix C). Note that the asymptotic decay at large separation of both the excess concentration and the charge density is given by the inverse decay length $\min \left(\lambda_{1}, \lambda_{2}\right)$, while in the case of the hydrophilic salt the decay length of the excess concentration is $\xi_{0}$, and the decay length of the charge is $\lambda_{D}^{*}$. This difference follows from the coupling of the concentration and charge fluctuations already in the Gaussian approximation in the case of the antagonistic salt.

The two decay lengths approach $\xi_{0}$ and $\lambda_{D}^{*}$ only far away from the disorder line, i.e. for $\lambda_{D}^{*} / \xi_{0} \gg\left(\sqrt{a}_{N}+\sqrt{a}_{D}\right)$ (low ionic strength, away from the critical point). In the limit of 
$\xi_{0} / \lambda_{D}^{*} \rightarrow 0$ Eqs.(48) and (49) take the forms

$$
\Phi(z) \simeq h_{0} e^{-z / \xi_{0}}
$$

and

$$
\rho_{q}^{*}(z) \simeq-\frac{\sigma_{0}}{\lambda_{D}^{*}} e^{-z / \lambda_{D}^{*}}
$$

where we took into account that $a_{D} \approx 1$.

The difference between the two decay lengths decreases when the disorder line $\left(\lambda_{D}^{*} / \xi_{0}=\right.$ $\left.\left(\sqrt{a}_{N}+\sqrt{a}_{D}\right)\right)$ is approached, and at the disorder line they become identical,

$$
\lambda_{1}^{2}=\lambda_{2}^{2}=\frac{\sqrt{a}_{D}}{\lambda_{D}^{*} \xi_{0}}
$$

For $\left(\sqrt{a}_{N}-\sqrt{a}_{D}\right)<\lambda_{D}^{*} / \xi_{0}<\left(\sqrt{a}_{N}+\sqrt{a}_{D}\right)$ Eq.(48) can be written inthe form

$$
\Phi(z)=A \cos \left(\lambda_{i m} z+\theta\right) e^{-\lambda_{r e} z}
$$

with similar damped oscillatory decay of $\rho_{q}^{*}(z)$, because $\lambda_{1}=\lambda_{r e}+i \lambda_{i m}$ and $\lambda_{2}=\lambda_{r e}-i \lambda_{i m}$ are complex conjugate numbers.

\section{The structure and effective potential between parallel surfaces}

We consider two selective and charged surfaces $\left(h_{0}, \sigma_{0}\right.$ and $\left.h_{L}, \sigma_{L}\right)$ which are separated by the distance L. We limit ourselves to identical surfaces, with $h=h_{0}=h_{L}$ and $\sigma=\sigma_{0}=\sigma_{L}$. The local concentration has the form

$$
\Phi(z)=\mathcal{A}_{1}\left[e^{-\lambda_{1} z}+e^{-\lambda_{1}(L-z)}\right]+\mathcal{A}_{2}\left[e^{-\lambda_{2} z}+e^{-\lambda_{2}(L-z)}\right]
$$

in the case of the structureless fluid, and

$$
\Phi(z)=\mathcal{A}\left[\cos \left(\lambda_{i m} z+\vartheta\right) e^{-\lambda_{r e} z}+\cos \left(\lambda_{i m}(L-z)+\vartheta\right) e^{-\lambda_{r e}(L-z)}\right]
$$

in the presence of mesoscopic inhomogeneities. The expressions for the amplitudes and the phase are rather long and will not be given here. The results of the linear theory for the excess concentration profiles, Eqs.(54) and (55), are compared with the numerical solutions of the full EL equations, Eqs. (62)-(64) and (4) for $T^{*}=6$ in Figs. 1,4,

As Fig. [1 shows, for small surface charges, surface fields and ion concentrations, and for temperatures far from the critical point, the linear theory agrees very well with the 
numerical solutions of the EL equations. Figs. 2 and 3 show the appearance of the periodic structure upon approaching the critical point and upon increasing $\lambda_{D}^{*}$. Finally, Fig. 4 indicates that for bigger surface charges and surface fields the linear theory differs significantly from the numerical results, however the qualitative agreement is preserved.
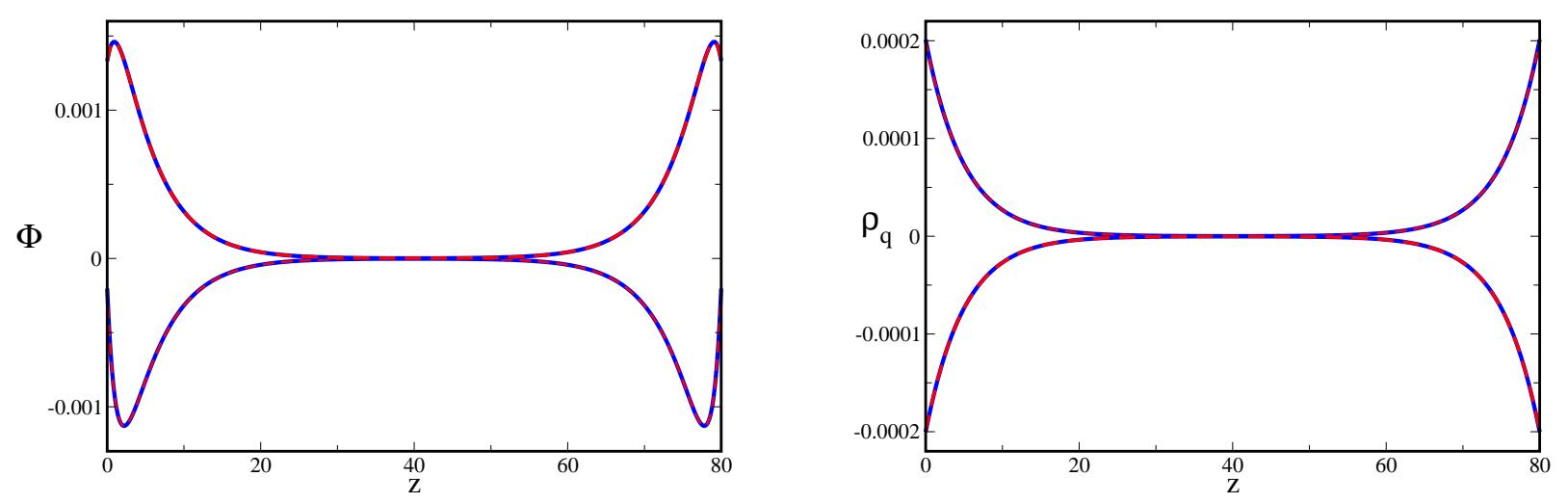

FIG. 1: The dimensionless concentration profile defined in Eq. 10 (left) and the dimensionless charge density (right). Solid lines correspond to numerical results of the full EL equations Eqs. (62)(64) and (44), while dash lines show analytical results (Eqs. (48), (49)). Dimensionless parameters are $\lambda_{D}^{*}=5,\left|T / T_{c}-1\right|=0.1, \bar{\rho}_{i o n}^{*}=0.001, h=0.001, \sigma=-0.001$ for top curves and $\sigma=0.001$ for bottom curves. The distance from the left wall $z$ is in units of the microscopic length $a \approx 0.4 \mathrm{~nm}$.

The excess grand potential (14) consists of the surface tension contribution, $\left(\gamma_{0}+\gamma_{L}\right) A^{*}$, and the effective potential between the confining surfaces of the dimensionless area $A^{*}$, $A^{*} \Psi(L)$. When the concentration $\Phi$ and the charge density $\rho_{q}^{*}$ satisfy the EL equations (67) and (66), then

$$
\begin{array}{r}
\gamma_{0}+\gamma_{L}+\Psi(L)=-\frac{J}{2}\left[\left(h_{0}-\frac{\sigma_{0} T^{*}}{1-\bar{\rho}_{i o n}^{*}}\right) \Phi(0)+\left(h_{L}-\frac{\sigma_{L} T^{*}}{1-\bar{\rho}_{i o n}^{*}}\right) \Phi(L)\right. \\
\left.+\frac{T^{*}}{\bar{\rho}_{\text {ion }}^{*}\left(1-\bar{\rho}_{i o n}^{*}\right)}\left(\sigma_{0} \rho_{q}(0)+\sigma_{L} \rho_{q}(L)\right)\right] .
\end{array}
$$

The results of the previous section give us for $\lambda_{D}^{*} / \xi_{0}>\left(\sqrt{a}_{N}+\sqrt{a}_{D}\right)$

$$
\Psi(L)=C_{1} e^{-\lambda_{1} L}+C_{2} e^{-\lambda_{2} L}
$$




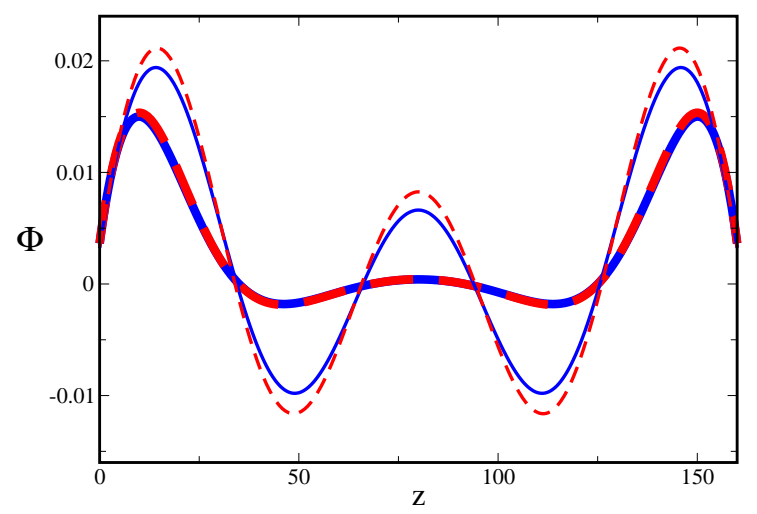

FIG. 2: The dimensionless concentration profile. Solid lines correspond to numerical results of the full EL equations Eqs. (62)-(64) and (44), while dash lines show analytical results, Eq. 48 The dimensionless parameters are $h=0.001, \sigma=-0.001, \lambda_{D}^{*}=10, \bar{\rho}_{i o n}^{*}=0.005$, (thin curves) $\left|T / T_{c}-1\right|=0.002$, and (thick curves) $\left|T / T_{c}-1\right|=0.005$. The distance from the left wall $z$ is in units of the microscopic length $a \approx 0.4 \mathrm{~nm}$.

and for $\left(\sqrt{a}_{N}-\sqrt{a}_{D}\right)<\lambda_{D}^{*} / \xi_{0}<\left(\sqrt{a}_{N}+\sqrt{a}_{D}\right)$ the above can be written in the form

$$
\Psi(L)=C \cos \left(\lambda_{i m} L+\theta\right) e^{-\lambda_{r e} L}
$$

where the expressions for the amplitudes are too long to be given here.

In Figs. 5 5 6 the effective potential per microscopic area $a^{2}$ between identical surfaces, obtained in the linearized and the nonlinear theory is presented for different $\lambda_{D}^{*}, T^{*}$ and different surface charge and selectivity of the surfaces. Fig. 5 presents a very good agreement at large distances between the linear theory and the full EL equations for small surface fields and surface charge, and for temperatures far from the critical point. When the critical point of the binary mixture is approached and the disorder surface is crossed, an oscillatory force between the surfaces is seen (Fig. 6 (right)). Note that for all the considered cases there is qualitative agreement between the linear theory and the numerical results.

The potential between surfaces of area $400 \mathrm{~nm} \times 400 \mathrm{~nm} \approx 10^{6} \mathrm{a}^{2}$ is $10^{6} \Psi(\mathrm{L})$. Note that for such a mesoscopic surface the first two extrema in Fig 6 are both of order of $k_{B} T$. 


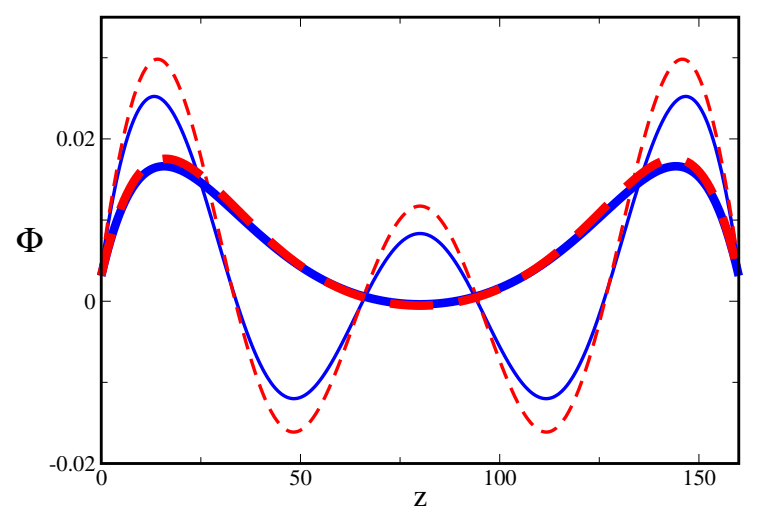

FIG. 3: The dimensionless concentration profile. Solid lines correspond to numerical results of the full EL equations Eqs. (62)-(64) and (44), while dash lines show analytical results, Eq. 48, The dimensionless parameters are $h=0.001, \sigma=-0.001,\left|T / T_{c}-1\right|=0.001$, (thick curves) $\lambda_{D}^{*}=20$, $\bar{\rho}_{i o n}^{*}=0.00125$, and (thin curves) $\lambda_{D}^{*}=10, \bar{\rho}_{i o n}^{*}=0.005$. The distance from the left wall $z$ is in units of the microscopic length $a \approx 0.4 \mathrm{~nm}$.

\section{THE CASE OF EXPERIMENTALLY STUDIED SAMPLES}

In this section we apply our theory to the systems studied experimentally in Ref.[2,

3, 5]. In the experiments the antagonistic salt sodium tetraphenylborate $\left(\mathrm{NaBPh}_{4}\right)$ was added to the 3-methylpyridine (3MP) and heavy water mixture near its lower critical point (LCP). Small-angle neutron scattering (SANS) was performed and a periodic structure with a periodicity of about $10 \mathrm{~nm}$ was reported in the experiment. The structure factor of the ternary mixture was determined for a few salt concentrations and a few temperatures. The structure factor was fitted to the expression obtained by Onuki and Kitamura [6] that has the same $k$ dependence as our Eq.(21).

Our theory has been developed for the upper critical point. In order to apply it to the system that phase separates for increasing temperature, we assume that in the coarse-grained description of the considered mixture the interaction parameter $J$ depends on $T$ in such a way that near the LCP $T^{*}=k_{B} T / J(T)$ decreases for increasing $T$. We shall not attempt to reproduce the phase diagram. Our purpose is a calculation of the effective potential between the confining surfaces for the samples studied experimentally in Refs. [2, 3, 5]. In order to 

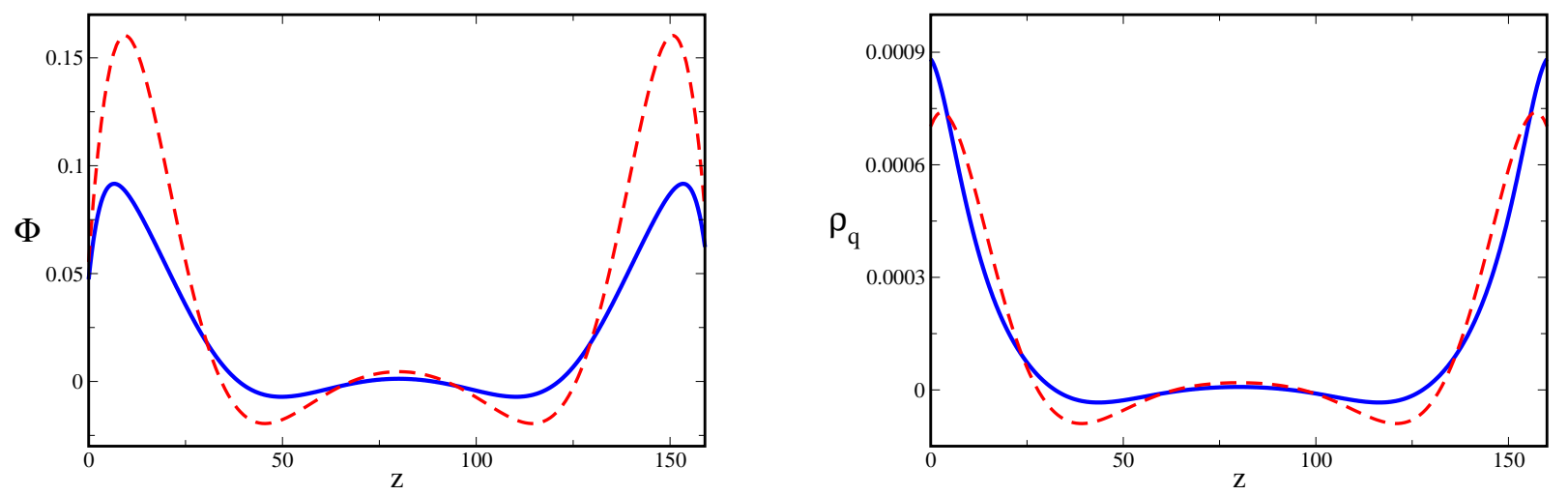

FIG. 4: The dimensionless concentration profile (left) and the dimensionless charge density (right). The dimensionless parameters are $h=0.03, \sigma=-0.01, \lambda_{D}^{*}=10, \bar{\rho}_{\text {ion }}^{*}=0.005$ and $\left|T / T_{c}-1\right|=$ 0.002. The distance from the left wall $z$ is in units of the microscopic length $a \approx 0.4 n m$.

model the particular samples, we assume that $a=0.4 n m, \bar{\Phi}=0$ for the samples with near-critical composition, $\lambda_{D}^{*}$ is given in Eq.(18) and we fit Eqs.(21)-(23) to the measured scattering intensity $S(k)$, assuming that $S(k)=S_{0} \tilde{G}(k)$. The remaining parameters are taken from Ref.[2, 5]. The selected samples and the parameters obtained from the fitting are given in Table I, and the fitting of the formulas (21)-(23) to the experimental curves is shown in Fig:7.

The excess concentration profiles and the effective potential for the samples studied in Ref.[5] and in Ref. [2] are shown in Figs. 8 and 9 respectively. We plot the results of the full EL equations, (62)-(64) and (44), between two identical weakly selective and weakly charged surfaces, for the parameters given in Table. I To obtain $\left|T / T_{c}-1\right|$ from $\xi_{0}$ we use the critical exponent $\nu=0.63$ instead of $1 / 2$ in Eq.(19). The structure factor takes a maximum for $k=k_{0}>0$ for three of the selected samples, and for $k_{0}=0$ for the low salt concentration, $\rho_{i o n}=1 \mathrm{mmol} / L$. For $k_{0}>0$ the exponentially damped oscillatory decay of correlations occurs, but the decay length is short compared to the period of oscillations (table I). For this reason the second extremum is much smaller than the first one, and is not seen on the plot. In the $k_{0}=0$ case the decay of correlations is given by two exponential functions. 


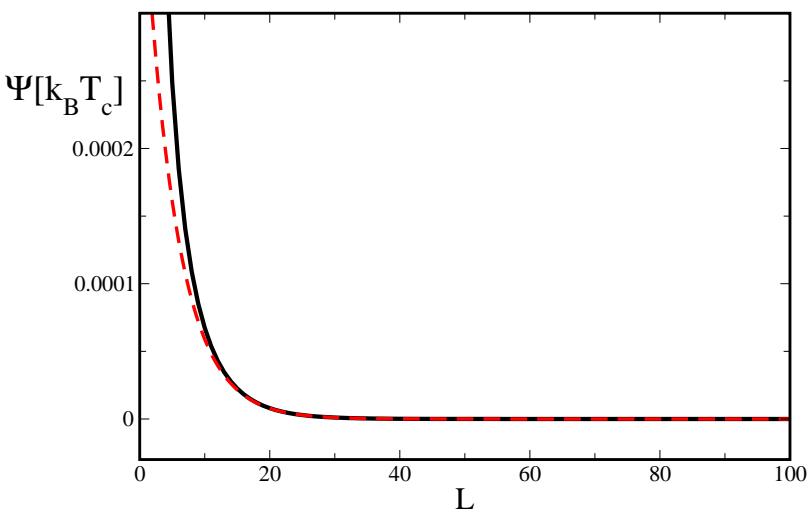

FIG. 5: The effective potential per microscopic area $a^{2}$ between two identical surfaces. Solid line corresponds to numerical solutions of the full EL equations Eqs. (62)-(64) and (44) and dash line corresponds to Eq.(56). The dimensionless parameters are $h=0.001, \sigma=0.001, \lambda_{D}^{*}=5$, $\left|T / T_{c}-1\right|=0.1$ and $\bar{\rho}_{i o n}^{*}=0.001$.

Note the change of the shape of $\Psi(L)$ with increased amount of salt (Fig 9). Because of the very short screening length the electrostatic repulsion that dominates in the $1 \mathrm{mM} / L$ sample is suppressed. In the $300 \mathrm{mM} / L$ sample a repulsion barrier $\sim 0.6 k_{B} T$ for surfaces of area $1.6 \cdot 10^{5} \mathrm{~nm}^{2}$ at the separation $L \approx 5 \mathrm{~nm}$ occurs, and the barrier is followed by a strong attraction for $L<3 \mathrm{~nm}$. For the intermediate salt concentration, $\bar{\rho}_{i o n}=7.2 \mathrm{mM} / \mathrm{L}$ a minimum of the potential of the depth $\sim 0.5 k_{B} T$ for surfaces of area $1.6 \cdot 10^{5} \mathrm{~nm}^{2}$ occurs for $L \simeq 20 \mathrm{~nm}$.

\section{DISCUSSION AND SUMMARY}

We have developed a Landau-type functional for a near-critical mixture with addition of antagonistic salt. We used the same coarse-graining procedure as in the case of the inorganic salt [10, 11, 30]. In both cases we have postulated that the OP for the phase separation, $\Phi$, is the concentration difference between the inorganic and organic components. While in the case of the inorganic salt $\Phi=\rho_{w}^{*}-\rho_{l}^{*}+\rho_{i o n}^{*}$, in the case of the antagonistic salt $\Phi=\rho_{w}^{*}-\rho_{l}^{*}+\rho_{q}^{*}$. Since there are two types of ions, we have postulated that the entropy has a form of the ideal entropy of mixing of a four-component mixture. The entropy of 

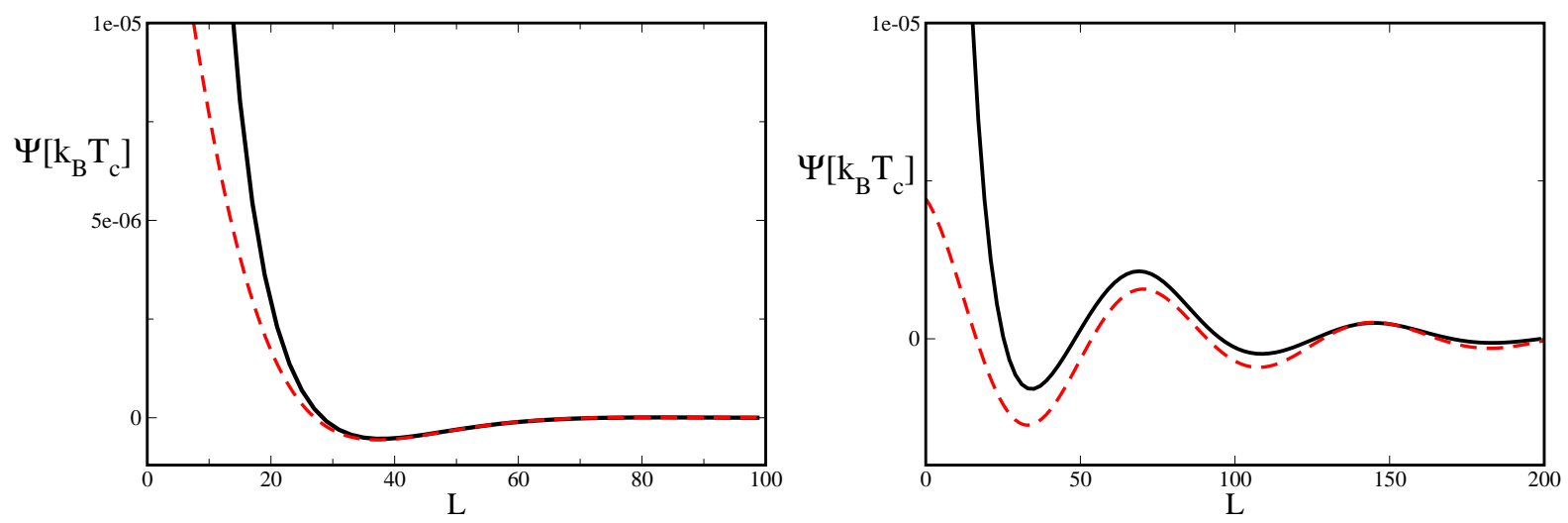

FIG. 6: The effective potential per microscopic area $a^{2}$ between two identical surfaces. Solid line corresponds to numerical solutions of the full EL equations Eqs. (62)-(64) and (44) and dash line corresponds to Eq.(56). The dimensionless parameters are $h=0.001, \sigma=-0.001, \lambda_{D}^{*}=15$, $\bar{\rho}_{\text {ion }}^{*}=0.005$ and (Left) $\left|T / T_{c}-1\right|=0.005$ and (right) $\left|T / T_{c}-1\right|=0.002$.

mixing has quite different form for the two different OP when expressed in terms of $\Phi, \rho_{\text {ion }}^{*}$ and $\rho_{q}^{*}$. As a consequence, the linearized EL equations for $\Phi$ and $\rho_{q}^{*}$ are coupled in the case of the antagonistic salt, and decoupled in the case of the inorganic salt. In the absence of the coupling of the linearized EL equations the decay lengths of $\Phi$ and $\rho_{q}^{*}$ are $\xi_{0}$ and $\lambda_{D}^{*}$ respectively. In the presence of the coupling the decay of both $\Phi(z)$ and $\rho_{q}^{*}(z)$ is given by the decay lengths $1 / \lambda_{1}, 1 / \lambda_{2}$ that differ from $\xi_{0}$ and $\lambda_{D}^{*}$. Another important consequence of the above coupling between $\Phi$ and $\rho_{q}^{*}$ is the lack of a qualitative difference between the solutions of the linearized and nonlinear EL equations. On the other hand, in our theory for the inorganic salt qualitatively different results in the linearized and nonlinear theories have been obtained [11, 29].

We should note that our theory is similar to the theory developed by Onuki and Kitamura [6] and studied in Ref.[16]. The main difference between the two theories is the OP of the phase separation. In Refs. [6, 16] the OP is identified with the concentration in the binary mixture, $\rho_{w}^{*}-\rho_{l}^{*}$, while we define the OP in Eq.(10). We take into account the van der Waals interactions between the ions, and consider the entropy of the four-component mixture, instead of a sum of the entropies of the two 2-component subsystems. Important 

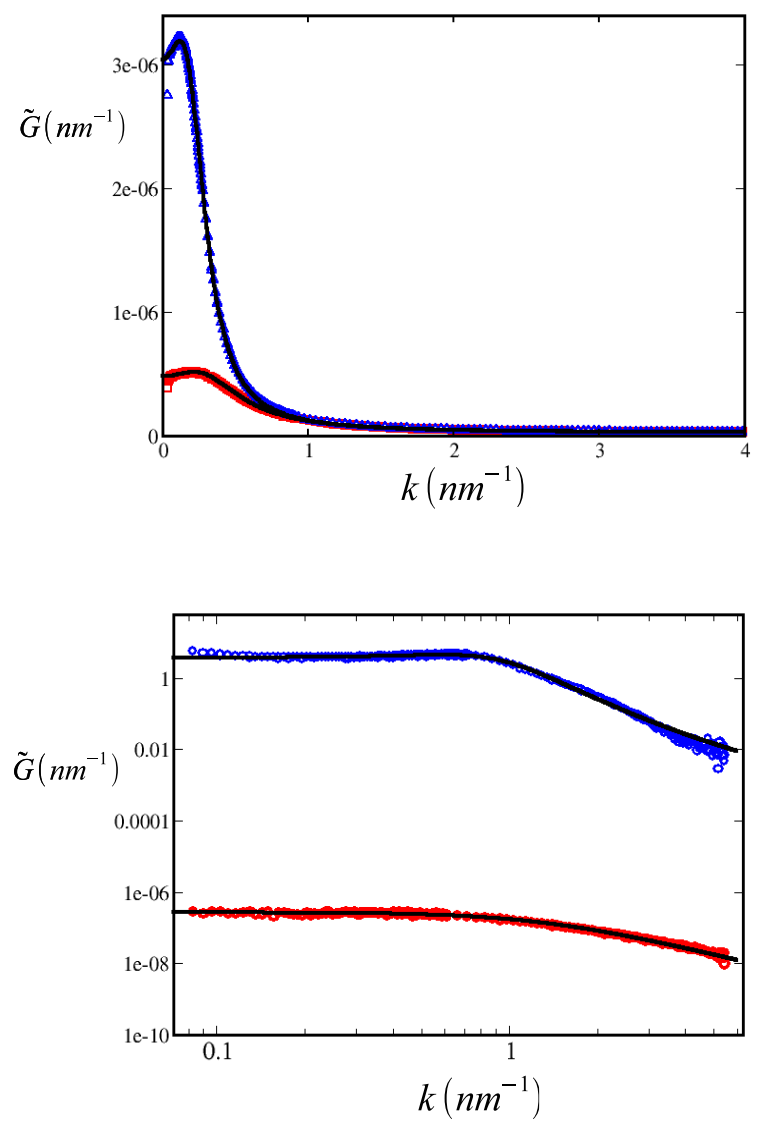

FIG. 7: SANS intensity measurements for near-critical composition of the $3 \mathrm{MP}, \mathrm{D}_{2} \mathrm{O}$ and $\mathrm{NaBPh}_{4}$ mixture. Top: $T=293 K$ (bottom curve), $T=313 K$ (top curve) and $\rho_{\text {ion }}=7.2 m M / L[5]$. Bottom: $T=280 K, \rho_{i o n}=300 \mathrm{mM} / L$ (top curve), and $\bar{\rho}_{i o n}=1 \mathrm{mM} / L$ (bottom curve)[2]. The remaining parameters are shown in Table I. The solid lines are the theoretical prediction of the correlation function, Eq.(21). The fit for $\bar{\rho}_{i o n}=300 \mathrm{mM} / L$ is better than in Ref.[2] because we take into account the dependence of $a_{D}$ on $\bar{\rho}_{i o n}$.

advantage of our approach is the link between the mesoscopic and the microscopic description that was a starting point of our derivation. Thanks to this link our theory is justified on a more fundamental level. However, in the case of the antagonistic salt the difference between our theory and the theory of Onuki and Kitamura is less significant than in the case of the inorganic salt. In the latter case the nonlinear coupling between $\Phi$ and $\rho_{q}^{*}$ plays a crucial role in our theory, and is ignored in Ref. [6, [16].

The effect of very small amount of solute should be independent of its kind. Our func- 


\begin{tabular}{lllllllll}
\hline$T(K)$ & $\rho_{\text {ion }}(m M / L)$ & $\lambda_{D}(n m)$ & $\xi_{0}(n m)$ & $a_{N}$ & $S_{0} \tilde{G}_{\Phi \Phi}(0)$ & $T^{*}$ & $\Lambda_{1}(n m)$ & $\Lambda_{2}(n m)$ \\
\hline 313 & 7.2 & 2.6 & 5.82 & 1.20 & $1.42 \cdot 10^{-8}$ & 106 & 5.5 & 34.6 \\
293 & 7.2 & 2.6 & 2.16 & 1.73 & $1.55 \cdot 10^{-8}$ & 152 & 2.94 & 25.1 \\
\hline 280 & 300 & 0.3 & 3.89 & 1.05 & 0.03875 & 160 & 1.90 & 8.26 \\
280 & 1 & 5.2 & 0.75 & 1.03 & $7.59 \cdot 10^{-8}$ & 160 & 0.76 & 5.16 \\
\hline
\end{tabular}

TABLE I: Parameters characterizing the samples studied in Ref.[5] (the first two rows) and in Ref.[2] (the last two rows). The data in the first three columns are taken or computed from the data given in Refs. [2, 5]. The data in the columns 4-6 are obtained from the fitting of the SANS intensity to the correlation function $\tilde{G}$ given in Eq.(21)-(23). The data in column 7 are computed from Eq.(22). For the first three rows the columns 8 and 9 show the decay length and the period of the damped oscillatory decay, i.e. $\Lambda_{1}=1 / \lambda_{r e}$ and $\Lambda_{2}=2 \pi / \lambda_{i m}$ (Eq,73). In the last row (1 $\mathrm{mM} / \mathrm{L}$ sample) the two decay lengths of the double exponential decay are shown, i.e. $\Lambda_{i}=1 / \lambda_{i}$ (see Eq.(40)).

tionals for the inorganic and antagonistic salt, however, differ significantly from each other. Nevertheless, we have verified that for small amount of ions and not very close to the critical point (for example for $\rho_{i o n}^{*}=0.001$ and $\left|T / T_{c}-1\right|=0.005$ ) the effective potential between parallel external surfaces has essentially the same form for the inorganic and antagonistic salt. For larger $\rho_{i o n}^{*}$ a quantitative difference between $\Psi(L)$ in the presence of the inorganic or the antagonistic salt can be seen (Fig,10). Qualitatively different shapes of $\Psi(L)$ are obtained if the correlation length is sufficiently large compared to the period of the concentration oscillations. The same amount of antagonistic salt leads to a deeper minimum of the effective potential at shorter separation than in the case of the inorganic salt, and the repulsion barrier occurs. This shape of the potential may occur when $\rho_{\text {ion }}^{*}$ is big enough, and the critical point is approached (Fig[10).

We have obtained a mesoscopic functional of the Landau-Brazovskii form (31) from a microscopic density-functional theory for a four-component mixture by a systematic coarsegraining procedure. A functional of the same form was successfully applied to amphiphilic systems [12, 13]. Thus, our theoretical result and the experimental observations of similar- 

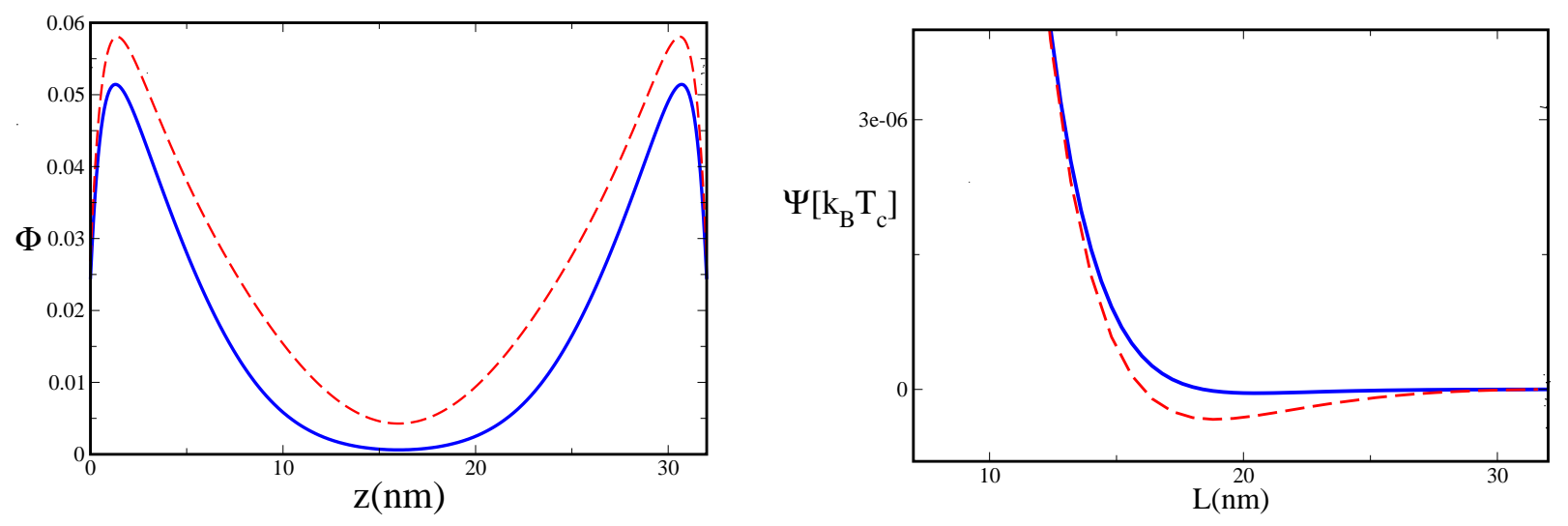

FIG. 8: (Left) Excess concentration profile (dimensionless) and (right) the effective potential per microscopic area $a^{2}$ (with $a \approx 0.4 n m$ ) resulted from full EL equations, (62)-(64) and (44), as a function of the distance between two identical surfaces. The parameters are selected according to experiment [5] for $7.12 \mathrm{mM} / \mathrm{L}$ of $\mathrm{NaBPh}_{4}$ added to $\mathrm{D}_{2} \mathrm{O}$ and metylpyridine at $313 \mathrm{~K}$ (dash line) and $293 K$ (solid line) given in Table. I The dimensionless selectivity and surface charge are $h=0.001$ and $\sigma=0.001$ respectively.

ity between the mesoscopic structure induced by amphiphiles and antagonistic salt [2] are complementary. We have limited to the Gaussian approximation here. It is well known that beyond the Gaussian approximation the fluctuations with the wavelength $k \simeq k_{0}$ yield a significant contribution to the correlation function, especially close to $t_{0}=0$. The $k$ dependence, however, is not changed up to a small correction; only $t_{0}$ is renormalized in the 1-loop approximation. We fitted our expression for $\tilde{G}(k)$ to the experimental curves. Since the decay of the OP and the shape of the effective potential are determined by the correlation function, in our results for $\Psi$ the renormalization of the model parameters is partially taken into account. This way we avoid the overestimation of the mesoscopic structure typical for the men-field results.

We have calculated the effective potential for 4 samples investigated experimentally in Ref.[2, [5]. The maximum of the structure factor occurs for $k_{0}>0$ for three of these samples. The oscillatory concentration and effective potential can be seen only in the sample with the largest concentration of ions, however. This is because the period of oscillations, $2 \pi / \lambda_{i m}$ is large compared to the decay length $1 / \lambda_{\text {re }}$ (Table I), whereas a detectable oscillatory potential can be expected for $1 / \lambda_{r e}>2 \pi / \lambda_{i m}$. Based on our results (Fig9) and on the analogy with surfactant mixtures where such forces were measured [14], we may expect oscillatory effective 

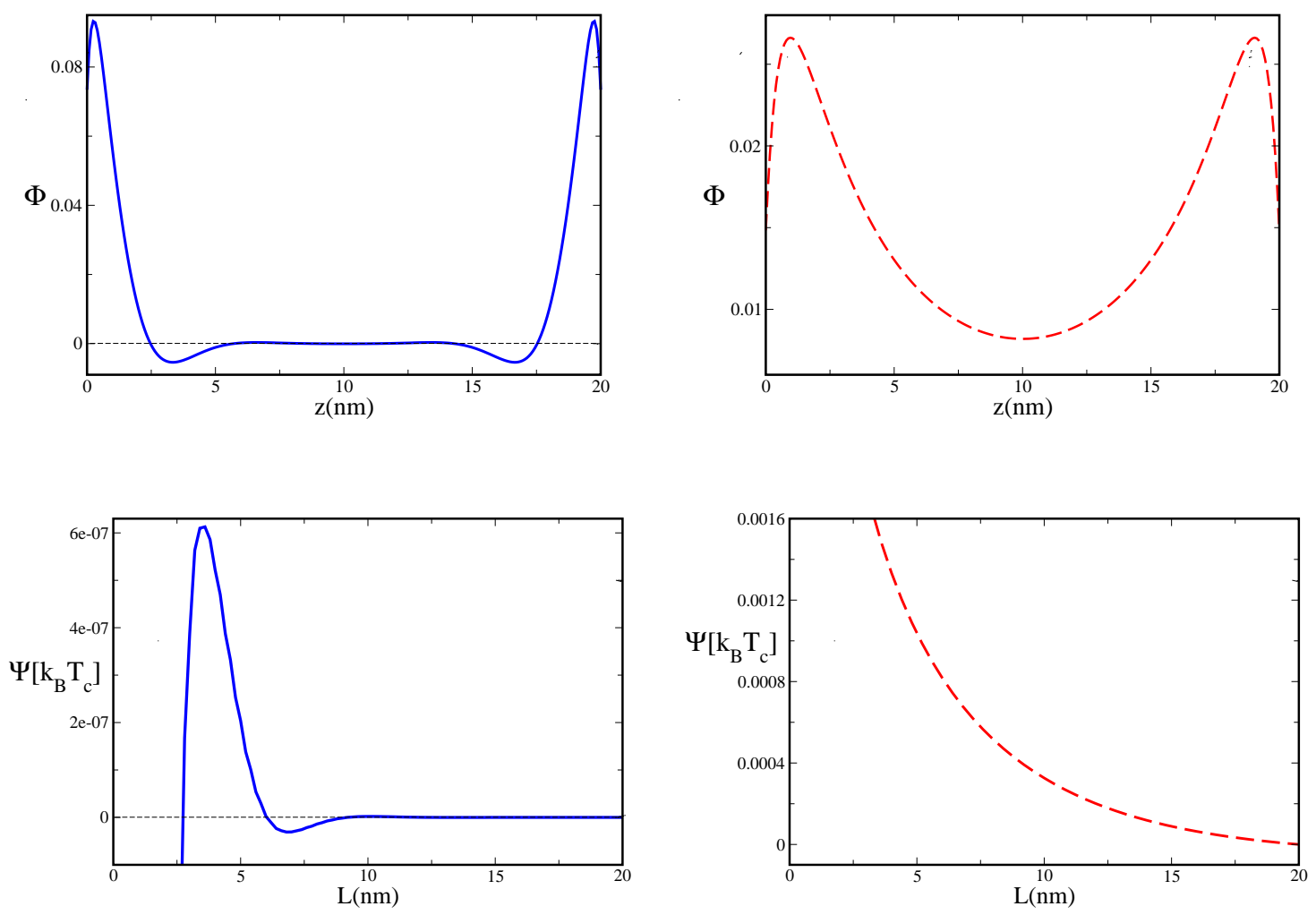

FIG. 9: (Top row) Excess concentration profiles (dimensionless) and (bottom row) the effective potential per microscopic area $a^{2}$ (with $a \approx 0.4 n m$ ) resulted from full EL equations, (62)- (64) and (4), as a function of the distance between two identical surfaces. The parameters are selected according to experiment [2] for the $300 \mathrm{mM} / L$ (solid lines) and $1 \mathrm{mM} / L$ (dash lines) of $N a B P h_{4}$ added to $D_{2} \mathrm{O}$ and metylpyridine sample at $T=280 \mathrm{~K}$ [2], given in Table. [1 The dimensionless selectivity and surface charge are $h=0.001$ and $\sigma=0.001$ respectively.

potential between the confining surfaces closer to the critical point or near the transition to the lamellar phase.

The potential in Figs 9 and 10 is attractive at short separations and repulsive at larger separations (SALR). For the SALR-type potentials between colloid particles finite clusters are expected, because the barrier prevents the clusters from further growth [32, 37].

We conclude that effective interactions between weakly charged colloid particles immersed in a near-critical mixture with antagonistic salt can exhibit very rich behavior. As a result, the particles can form different types of structures. Small changes of the amount of ions or temperature can lead to qualitative changes of the interactions between the particles. The sensitivity of the effective interactions to the thermodynamic state is a property that may 

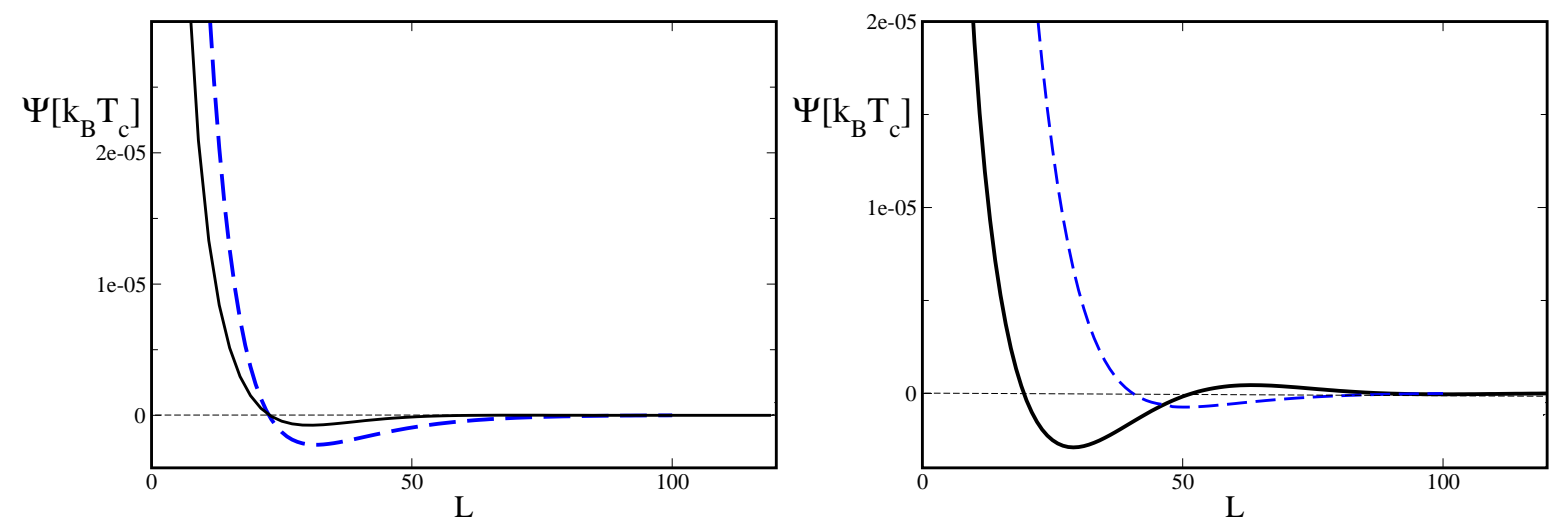

FIG. 10: Effective potential obtained from numerical solutions of the full EL equations. Solid lines correspond to the current theory while the dash lines show the results of a theory of hydrophilic ions [11, 30]. The parameters are $h=0.001, \sigma=-0.001, \lambda_{D}^{*}=10, \bar{\rho}_{i o n}^{*}=0.005$, (left) $\left|T / T_{c}-1\right|=$ 0.005, (right) $\left|T / T_{c}-1\right|=0.002$. $L$ is in units of the microscopic distance $a=0.4 \mathrm{~nm}$.

allow for manipulating with the structure of colloids.

\section{Acknowledgments}

We thank Dr. Koichiro Sadakane and Dr. Jan Leys for providing us the data with their experimental structure factor. The work of FP was realized within the International PhD Projects Programme of the Foundation for Polish Science, cofinanced from European Regional Development Fund within Innovative Economy Operational Programme "Grants for innovation". AC acknowledges the financial support by the NCN grant 2012/05/B/ST3/03302.

\section{APPENDICES}

\section{A. The Euler-Lagrange equations}

From $\delta \Omega\left(\Phi(\mathbf{r}), \rho_{q}^{*}(\mathbf{r}), \rho_{\text {ion }}(\mathbf{r})\right) / \delta \rho_{\text {ion }}^{*}(\mathbf{r})=0$ we obtain

$$
\mu_{i o n}=\frac{T^{*}}{2} \ln \left(\frac{\rho_{i o n}^{* 2}(\mathbf{r})-\rho_{q}^{* 2}(\mathbf{r})}{\left(1-\rho_{i o n}^{*}(\mathbf{r})\right)^{2}-\left(\Phi(\mathbf{r})-\rho_{q}^{*}(\mathbf{r})\right)^{2}}\right) .
$$

In a uniform fluid Eq.(59) takes the form

$$
\mu_{i o n}=T^{*} \ln R
$$


where

$$
R^{2}=\frac{\bar{\rho}_{i o n}^{* 2}}{\left(1-\bar{\rho}_{i o n}\right)^{* 2}-\bar{\Phi}^{2}} .
$$

By equating RHS of Eqs. (60) and (59) we obtain

$$
\rho_{\text {ion }}^{*}(\mathbf{r})=\frac{-R^{2}+\sqrt{R^{2}-\left(1-R^{2}\right)\left[R^{2}\left(\Phi(\mathbf{r})-\rho_{q}^{*}(\mathbf{r})\right)^{2}-\rho_{q}^{*}(\mathbf{r})^{2}\right]}}{1-R^{2}} .
$$

With the help of Eq. (62) we can eliminate $\rho_{i o n}^{*}(\mathbf{r})$ from Eq. (12). The remaining EL equations are obtained in a similar way, and have the forms

$$
e \beta \psi_{e l}(\mathbf{r})+\frac{1}{2} \ln \left[\frac{\left(\rho_{i o n}^{*}(\mathbf{r})+\rho_{q}^{*}(\mathbf{r})\right)(1-\Phi(\mathbf{r}))+\left(\rho_{q}^{*}(\mathbf{r})^{2}-\rho_{i o n}^{*}(\mathbf{r})^{2}\right)}{\left(\rho_{i o n}^{*}(\mathbf{r})-\rho_{q}^{*}(\mathbf{r})\right)(1+\Phi(\mathbf{r}))+\left(\rho_{q}^{*}(\mathbf{r})^{2}-\rho_{i o n}^{*}(\mathbf{r})^{2}\right)}\right]=0
$$

and

$$
\frac{d^{2} \Phi(\mathbf{r})}{d \mathbf{r}^{2}}=-b \Phi(\mathbf{r})+\frac{T^{*}}{2} \ln \left[\frac{1-\rho_{i o n}^{*}(\mathbf{r})+\Phi(\mathbf{r})-\rho_{q}^{*}(\mathbf{r})}{1-\rho_{i o n}^{*}(\mathbf{r})-\Phi(\mathbf{r})+\rho_{q}^{*}(\mathbf{r})}\right] .
$$

Eqs. (62)-(64) and (44) form a closed set of two differential and two algebraic equations.

When $\rho_{q}^{*}(\mathbf{r}), \Phi$ and

$$
\vartheta(\mathbf{r})=\rho_{i o n}^{*}(\mathbf{r})-\bar{\rho}_{i o n}^{*}
$$

are small, then we can solve analytically the linearized EL equations. Here we focus on the critical composition, $\bar{\Phi}=0$. Let us limit ourselves to the functions that depend only on $z$. From (64) we obtain

$$
\frac{d^{2} \Phi(z)}{d z^{2}}=\xi_{0}^{-2} \Phi(z)-\frac{T^{*}}{\left(1-\bar{\rho}_{i o n}^{*}\right)} \rho_{q}^{*}(z)
$$

and from (63) and (41) we have

$$
\frac{d^{2} \rho_{q}^{*}(z)}{d z^{2}}=\kappa^{2}\left(1-\bar{\rho}_{i o n}^{*}\right) \rho_{q}^{*}(z)+\bar{\rho}_{i o n}^{*} \frac{d^{2} \Phi(z)}{d z^{2}} .
$$

\section{B. Internal energy in Fourier representation}

The electrostatic energy Eq. (33) in the Fourier representation is given by

$$
U_{e l}=\frac{1}{2} \int_{V} \frac{d \mathbf{k}}{(2 \pi)^{d}}\left(\frac{4 \pi e^{2}}{a \epsilon k^{2}}\right) \tilde{\rho}_{q}^{*}(\mathbf{k}) \tilde{\rho}_{q}^{*}(\mathbf{k})
$$

where the wavenumber $k$ is dimensionless (in $a^{-1}$-units) and we used the Poisson equation in Fourier representation

$$
k^{2} \tilde{\psi}_{e l}(\mathbf{k})=\frac{4 \pi e}{a \epsilon} \tilde{\rho}_{q}^{*}(\mathbf{k}) .
$$


The van der Waals contribution Eq. (9) in the Fourier representation takes the form

$$
U_{v d W}=\frac{J}{2} \int_{V} \frac{d \mathbf{k}}{(2 \pi)^{d}}\left(-b+k^{2}\right) \tilde{\Phi}(\mathbf{k}) \tilde{\Phi}(-\mathbf{k}) .
$$

\section{Parameters in the solutions of the EL equations}

The amplitudes in Eq.(48) are

$$
\left\{\begin{array}{l}
A_{1}=\frac{h_{0}\left(\lambda_{2}^{2}-\zeta^{-2}\right) \lambda_{2}-\frac{T^{*} \sigma_{0}}{\lambda_{D}^{* 2}}\left(1+\lambda_{2}\right)}{D}, \\
A_{2}=-\frac{h_{0}\left(\lambda_{1}^{2}-\zeta^{-2}\right) \lambda_{1}-\frac{T^{*} \sigma_{0}}{\lambda_{D}^{* 2}}\left(1+\lambda_{1}\right)}{D} .
\end{array}\right.
$$

with

$$
D=\left(\lambda_{1}-\lambda_{2}\right)\left[\zeta^{-2}-\lambda_{1}^{2}-\lambda_{2}^{2}-\lambda_{1} \lambda_{2}\left(1+\lambda_{1}+\lambda_{2}\right)\right]
$$

where $\lambda_{i}$ and $\zeta$ are given in Eq.(40) and (47) respectively. The real and imaginary parts of the inverse decay lengths $\lambda_{i}$ are

$$
\left\{\begin{array}{l}
\lambda_{r e}^{2}=\frac{1}{4 \lambda_{D}^{* 2}}\left[\left(\sqrt{a_{D}}+\frac{\lambda_{D}^{*}}{\xi_{0}}\right)^{2}-a_{N}\right], \\
\lambda_{i m}^{2}=\frac{1}{4 \lambda_{D}^{* 2}}\left[a_{N}-\left(\frac{\lambda_{D}^{*}}{\xi_{0}}-\sqrt{a_{D}}\right)^{2}\right] .
\end{array}\right.
$$

[1] V. Balevicius and H. Fuess, Phys. Chem. Chem. Phys. 1, 1507-1510 (1999).

[2] K. Sadakane, M. Nagao, H. Endo and H. Seto, J. Chem. Phys 139, 234905 (2013).

[3] K. Sadakane, A. Onuki, K. Nishida, S. Koizumi and H. Seto, Phys. Rev. Lett. 103, 167803 (2009).

[4] K. Sadakane, A. Onuki, K. Nishida, S. Koizumi and H. Seto, Soft Matter 7, 1334 (2011).

[5] J. Leys, D. Subramanian, E. Rodezno, B. Hammouda and M. A. Anisimov, Soft Matter 9, $9326(2013)$.

[6] A. Onuki and H. Kitamura, J. Chem. Phys 121, 3143 (2004).

[7] N. Lei, C. R. Safinya, D. Roux and K. S. Liang, Phys. Rev. E 56, 608 (1997).

[8] L. Porcar, W. A. Hamilton, P. D. Butler and G. G. Warr, Langmuir 19, 10779 (2003).

[9] F. Nallet, R. Laversanne and D. Roux, J. Phys. II 3, 487 (1993). 
[10] A. Ciach and A. Maciołek, Phys. Rev. E 81, 041127 (2010).

[11] F. Pousaneh, A. Ciach and A. Maciołek, Soft Matter 10, 740-783 (2014).

[12] G. Gompper and M. Schick, in Phase Transitions and Critical Phenomena, edited by C. Domb and J. L. Lebowitz (Academic, London, 1994), Vol. 16.

[13] A. Ciach and W. T. Góźdź, Annu. Rep. Prog. Chem., Sect.C 97, 269 (2001).

[14] D. A. Antelmi, P. Kékicheff and P. Richetti, Langmuir 15, 7774-7788 (1999).

[15] S. A. Brazovskii, Sov. Phys. JETP 41, 8 (1975).

[16] R. Okamoto and A. Onuki, Phys. Rev. E 84, 051401 (2011).

[17] C. Hertlein, L. Helden, A. Gambassi, S. Dietrich and C. Bechinger, Nature 451, 172 (2008).

[18] U. Nellen, J. Dietrich, L. Helden, S. Chodankar, K. Nygard, J. F. van der Veen and C. Bechinger, Soft Matter 7, 5360 (2011).

[19] A. Gambassi, A. Maciołek, C. Hertlein, U. Nellen, L. Helden and C. Bechinger and S. Dietrich, Phys. Rev. E 80, 061143 (2009).

[20] V. D. Nguyen, S. Faber, Z. Hu, G. H. Wegdam and P. Schall, Nature Communications 4, 1584 (2013).

[21] D. Bonn, J. Otwinowski, S. Sacanna, H. Guo, G. Wegdam and P. Schall, Phys. Rev. Lett. 103, 156101 (2009); A. Gambassi and S. Dietrich, Phys. Rev. Lett. 105, 059601 (2010)

[22] M. Krech, Phys. Rev. E. 56, 2 (1996).

[23] O. Vasilyev, A. Maciołek and S. Dietrich, Phys. Rev. E. 84, 041605 (2011).

[24] M. Krech, (World Scientific, Singapore, 1994).

[25] M. T. Dang, A. V. Verde, V. D. Nguyen, P. G. Bolhuis and P. Schall, J. Chem. Phys 139, 094903 (2013).

[26] F. Pousaneh, Effect of ionic solutes on thermodynamic Casimir force, PhD thesis, Institute of Physical Chemistry, Polish Academy of Sciences, Poland (2014).

[27] M. Bier, A. Gambassi, M. Oettel and S. Dietrich, Europhys. Lett. 95, 60001 (2011).

[28] S. Samin and Y. Tsori, J. Chem. Phys. 136, 154908 (2012).

[29] F. Pousaneh and A. Ciach, J. Phys. Condens. Matter 23, 41201 (2011).

[30] F. Pousaneh, A. Ciach and A. Maciołek, Soft Matter 8, 3567 (2012).

[31] J.-L. Barrat and J.-P. Hansen, Basic concepts for simple and complex liquids (Cambridge University Press, Cambridge, 2003).

[32] A. Ciach, J. Pękalski and W. T. Góźdź, Soft Matter 9, 6301 (2013). 
[33] R. Evans, Adv. Phys 28, 143 (1979).

[34] A. Ciach and G. Stell, J. Mol. Liq. 87, 255 (2000).

[35] L. Leibler, Macromolecules 13, 1602 (1980).

[36] W. T. Góźdź and R. Hołyst, Phys. Rev. E 54, 5012 (1996).

[37] A. Stradner, H. Sedgwick, F. Cardinaux, W.C.K. Poon, S.U. Egelhaaf and P. Schurtenberger, Nature 432, 492 (2004).

[38] D. G. Barci, L. Ribeiro and D. A. Stariolo, Phys. Rev. E 87, 062119 (2013). 